\title{
Data retention after a patient withdraws consent in clinical trials
}

\author{
This article was published in the following Dove Press journal: \\ Open Access Journal of Clinical Trials \\ 16 April 201 I \\ Number of times this article has been viewed
}

\author{
André P Gabriel \\ Charles P Mercado \\ Department of Medicine, \\ Columbia University Medical Center, \\ New York, NY, USA
}

\begin{abstract}
Patient retention is critically important in the conduct of a successful clinical trial. The power in numbers in multicenter trials is dependent on the completion of follow-up for every patient randomized. If at the end of a clinical trial, a significant number of randomized patients are missing outcome data, there will not be enough pool for data analyses to conclude a study based on its primary and secondary objectives. When patients who are either lost to follow-up or who withdraw consent during the clinical trial are eliminated from the data pool, they subsequently affect the power and the validity of conclusions derived from the clinical study. This paper aims to present current guidance on data retention for patients who have withdrawn consent from clinical trials.
\end{abstract}

Keywords: withdrawal of consent, patient follow-up, guidance

\section{Introduction}

Clinical trials are prospective studies that either test a new drug or a combination of drugs, or assess a new approach to treatment. These are conducted to determine the safety and efficacy of the intervention in a randomized human population. Ultimately, clinical trials pave the way towards the improvement of diagnosis and treatment of diseases by introducing novel interventions and therapies.

Essential to the conduct of a clinical trial is the randomization of a population subject to the study, recruitment of eligible participants based on inclusion and exclusion criteria, and the completion of participation of patients in the study. ${ }^{1}$ Hence clinical trials are expensive, labor-extensive, and time-consuming.

Withdrawal of patients from clinical studies is not an uncommon phenomenon and is inevitable in some circumstances. Lack of efficacy of the drug being tested, the occurrence of adverse and intolerable side effects, lack of patient follow-up, or simply patient withdrawal of consent during the clinical trial are some reasons encountered. ${ }^{2}$ Accordingly, the proportion of patients who have withdrawn consent, is one of the quality indicators for randomized controlled trials. ${ }^{3}$

Informed consent is defined as the process of learning the key facts about a clinical trial before deciding whether or not to participate in a study. ${ }^{4}$ It is a continuous and dynamic process in which participants should be reminded at every clinical visit for the duration of the clinical trial; hence it is important to maintain an open dialog between the physician and the participant. ${ }^{5}$

Recruited patients should be aware at the beginning that they can freely withdraw (discontinue participation) their informed consent at any time during the clinical
Correspondence: André P Gabrie Department of Medicine, Columbia University Medical Center, New York, NY, USA

$\mathrm{Tel}+\mathrm{I} 2123059282$

Fax + I 2123422904

Email apg2120@columbia.edu
Dovepress

DOI: $10.2147 /$ OAJCT.SI3960
Open Access Journal of Clinical Trials 201 I:3 I5-19

(c) 20II Gabriel and Mercado, publisher and licensee Dove Medical Press Ltd.This is an Open Access 
trial. ${ }^{6}$ In the same manner, the investigator can terminate a subject's participation in a research study without regard to the subject's consent. ${ }^{7}$ Among justifiable reasons for termination of participation by the investigator includes patient's inability to continue followups; hence, there is a certain degree of volatility of the informed consent.

For the purpose of delineating between dropouts versus withdrawal of consent in a clinical trial, we define 'withdrawal of consent' as the patient's voluntary termination of informed consent to participate in a clinical trial at any point during the conduct of the study. This definition covers several clauses and reasons as to why patients opt to withdraw consent and participation in a clinical trial (Figure 1). The most commonly encountered reasons include: 1) difficulty to comply with study medications; 2) adverse drug effects; 3 ) intolerable side effects; 4) physical debilitation to continue in-office visits; 5) patient reaching study endpoint; 6) hesitation to continue with study medications; and 7) relocation to another state or country, which makes it impossible for patients to followup.

Participation in a clinical trial can be classified to several levels. For some investigators, the desire of the patient to discontinue in the therapeutic interventional part of the trial constitutes a withdrawal of consent. ${ }^{8}$ However, it is appropriate to consider that these patients may have withdrawn consent for the medication, but have not

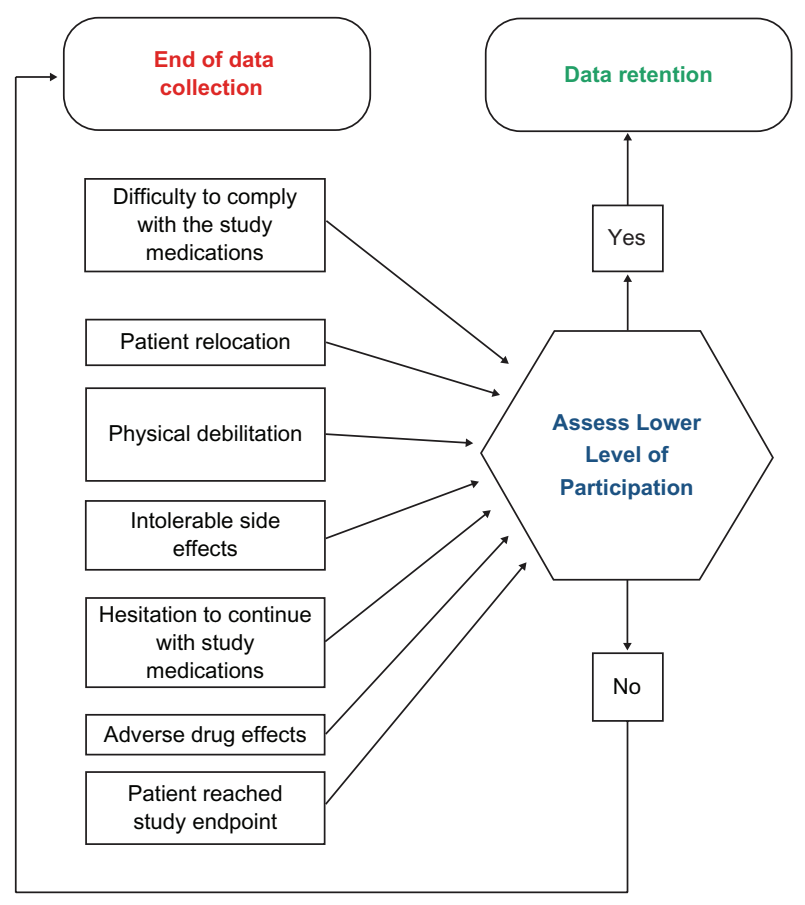

Figure I Most common reasons for study follow-up termination. withdrawn consent for participation in the clinical trial. As for patients who also refuse inhouse visits for study followup, investigators tend to interpret this as a withdrawal of consent. ${ }^{9}$ On the other hand, patients may be willing to be contacted by letter or telephone or other means of communication even though they are unwilling to attend further study visits. Such contacts can provide valuable information on survival status or study endpoints. In several instances, many of these withdrawals of consent can be offered lower levels of participation in the clinical trial. ${ }^{10}$ More often than not, both the investigators and patients are trapped in ambiguity with the nature of the withdrawal of informed consent and this is brought up only after questions are raised by study monitors about incomplete case report forms. It is therefore imperative for patients, investigators, clinicians, and regulatory bodies that additional information is provided whenever possible. Preferably, information should be provided by the patient or if not feasible, by the investigator. Additional information needs to be formulated to address specific questions regarding medications and followup in order to determine the extent by which the patient agrees to provide further data or be subjected to additional treatment. ${ }^{10}$

Another issue worth mentioning is what to do with a patient who has withdrawn consent and subsequently dies. In several clinical trials where investigators have sought an ethical and legal opinion, the consensus is that there is no right of confidentiality about death given that this is part of public records. Although data on morbidity may be lost in these patients, mortality data can still be obtained.

In this paper, we aim to review current guidelines on data retention for patients who have withdrawn consent from clinical trials. With the new guidelines recently released by the Office of Human Research Protections (OHRP), ${ }^{11}$ the relevance of this review is timely, which may serve principal investigators, biostatisticians, and research specialists in the proper analyses and treatment of data obtained in clinical trials.

\section{Discussion}

In September 2010, the Office of Human Research Protections (OHRP) released a guidance document entitled, "Guidance on Withdrawal of Subjects from Research: Data Retention and Other Related Issues". ${ }^{11}$ This came two years after the Food and Drug Administration (FDA) released a similar guidance document, "Guidance for Sponsors, Clinical Investigators, and Institutional Review Board (IRB): Data Retention When Subjects Withdraw from FDA-regulated clinical trials". 
These guidance documents highlight the significance of the informed consent and withdrawal of consent in clinical trials. They represent the first formal public guidance from the agencies and affirm previous informal statements and positions of FDA and OHRP regarding a subject who withdraws participation from clinical research by choice or who is withdrawn by the investigator. ${ }^{9}$

\section{FDA guidance}

The FDA's guidance is consistent with its longstanding policy for FDA-regulated trials that information collected prior to a subject's withdrawal should remain part of the study database to ensure the scientific validity of the research. ${ }^{1}$ The FDA guidance describes current FDA requirements for collection, maintenance, and submission to FDA of complete and accurate data regarding clinical investigations. It also articulated FDA's concerns relating to the reliability of study analyses that exclude data for withdrawn subjects; specifically, that withdrawn subjects are more likely to have experienced adverse events or a failure of efficacy, and the exclusion of their data has an increased probability of introducing bias and negatively impacting the scientific validity of the research.

The following key points were cited from the "Guidance for Sponsors, Clinical Investigators and IRBs, Data Retention When Subjects Withdraw from FDA-Regulated Clinical Trials": 1

1. According to the FDA regulations, when a subject withdraws from a study, the data collected on the subject to the point of withdrawal remains part of the study database and may not be removed.

2. An investigator may ask a subject who is withdrawing whether the subject wishes to provide continued follow-up and further data collection subsequent to their withdrawal from the interventional portion of the study. Under this circumstance, the discussion with the subject would distinguish between study related interventions and continued follow-up of associated clinical outcome information, such as medical course or laboratory results obtained through non-invasive chart review, and address the maintenance of privacy and confidentiality of the subject's information.

3. If the subject withdraws from the interventional portion of the study but agrees to continued follow-up of associated clinical outcome information as described in the previous section, the investigator must obtain the subject's informed consent for this limited participation in the study (assuming such a situation was not described in the original informed consent form). In accordance with FDA regulations, IRB approval of informed consent documents would be required. ${ }^{13}$

4. If a subject withdraws from the interventional portion of the study and does not consent to continued follow-up of associated clinical outcome information, the investigator must not access for purposes related to the study the subject's medical record or other confidential records requiring the subject's consent. However an investigator may review study data related to the subject prior to the subject's withdrawal from the study, and may consult public records, such as those establishing survival status.

\section{OHRP guidance}

The OHRP guidance describes the manner in which the Common Rule permits an investigator to continue to analyze previously collected data and in certain circumstances collect additional data regarding a subject who withdraws (or is withdrawn by the investigator) from a clinical trial. Regardless of whether a subject's withdrawal constitutes complete or partial discontinuation of participation in the research study, an investigator may continue to analyze data collected from the subject or derived from the subject's biological specimens up to the time of withdrawal without regard to the subject's consent, since OHRP has determined that these activities are not included in the definition of 'participation' in research requiring a subject's consent. ${ }^{11}$

While OHRP excludes analysis of previously collected data (as defined in the context of participation in research requiring informed consent), the OHRP guidance distinguishes the applicability of such activities based on the definition of "human subjects research" requiring IRB approval in contrast to the continuation of analysis of previously collected data after the cessation of patient treatment or intervention. As such, these activities require an annual IRB review. ${ }^{9}$

Each entity engaged in clinical research is responsible for ensuring the scientific validity, ethical soundness, and regulatory compliance of its operations. Entities subject to the FDA guidance and OHRP guidance should review and evaluate current operations, processes, policies, and compliance infrastructure to determine whether the recommendations set forth in these guidance documents are addressed adequately. Specifically, sponsors, investigators, institutions, IRBs, and other entities involved in clinical research should review policies and procedures, training 
materials, and template documents related to protocol development, statistical analysis, and informed consent of clinical research subjects to determine whether they comply with these new guidance documents' recommendations.

In applying these guidance documents in today's clinical trials, Dow and Goldstein ${ }^{9}$ have laid out the following recommendations:

1. Updating applicable policies and procedures to ensure consistency with the recommendations of FDA and OHRP, as applicable. These may include: policies related to statistical analysis of data from subjects who discontinue participation in a clinical trial; retention and analysis of biological specimens collected from withdrawn subjects; informed consent document development, review or completion; the informed consent process; development of protocol sections regarding the informed consent and discontinuation of study subjects; IRB review of initial protocols and informed consent documents; IRB continuing review reports and IRB continuing review of research activities following discontinuation of study subjects.

2. Reviewing the informed consent document templates to ensure accuracy of disclosure as to the extent by which subject data will be used in the event of subject withdrawal and should be clearly and explicitly stated in the informed consent documents.

3. Providing training to individuals involved in planning, review, implementation, and analysis of clinical trials regarding the recommendations of the FDA guidance and OHRP guidance, as applicable, and any updates to template documents, policies, processes, and procedures.

4. Submitting comments to address the uncertainties in the OHRP guidance or in the FDA guidance. One area for potential comment may include the lack of clarity regarding the treatment of biological specimens under the FDA guidance. While the FDA guidance is a final guidance document, issued for immediate implementation, the FDA will accept comments on such guidance documents.

Additionally, sponsors of clinical research should consider:

5. Reviewing protocol templates and case report forms to ensure these documents incorporate additional documentation recommendations for withdrawn subjects, such as reason for withdrawal and whether withdrawal was the decision of the subject or the investigator.

6. Confirming that statistical analysis plans include analysis of data for withdrawn subjects. While many sponsors may perform intent-to-treat analyses and have procedures in place to interpret or impute missing data from withdrawn subjects, confirmation that statistical analysis plans comply with the recommendations of the FDA guidance may be considered.

\section{Conclusion}

The FDA and OHRP guidance have parallel policies on addressing data retention after a patient withdraws consent in clinical trials. However, the FDA guidance went further by stating, "If a subject withdraws from the interventional portion of a study and does not consent to continued follow-up of associated clinical outcome information, the investigator must not access, for purposes related to the study, the subject's medical record or other confidential records requiring the subject's consent". However, an investigator may review the data collected prior to the subject's withdrawal from the study and may consult public records such as those establishing survival status, ${ }^{1}$ which is lacking in the OHRP guidance. The establishment of survival status is critical in large-scale multicenter clinical trials whose primary endpoint include death particularly in large multicenter cardiovascular clinical trials. Without sufficient patient retention from the time of study initiation to closeout, the number of remaining participants may prove to be too small for a pool from which a conclusive evidence is derived to prove or disprove the goal of the clinical sponsor. ${ }^{6}$ Although, the FDA and OHRP guidance have paved the way for regulatory understanding on data retention after patient withdrawal of consent, the best approach that investigators and site staff should employ should start at the time of the informed consent process. It is important that patients are well informed about their participation in the clinical trial and that the informed consent process be fully administered. Investigators, site staff, and patients should have an open communication throughout the duration of the trial. Patients who receive more qualitative/quantitative information about the trial in which they are participating are less likely to be lost to follow-up or withdraw their consent. ${ }^{4}$

\section{Acknowledgment}

The authors would like to thank Dr Ma-Am Joy R Tumulak for reviewing the manuscript and for her helpful discussion regarding this work.

\section{Disclosure}

The authors report no conflicts of interest in this work. 


\section{References}

1. US Department of Health and Human Services, FDA. Guidance for Sponsors, Clinical Investigators, and IRBs Data Retention When Subjects Withdraw from FDA-Regulated Clinical Trials. 2008 Oct. Available from: http://www.fda.gov/OHRMS/DOCKETS/98fr/FDA-2008-D0576-gdl.pdf. Accessed March 16, 2011.

2. Cleland J, Torp-Pedersen C, Coletta A, et al. A method to reduce loss to follw-up in clinical trials: informed, withdrawal of consent. Eur J Heart Failure. 2004;6:1-2.

3. Gordon E, Prohaska T. The ethics of withdrawal from study participation. Account Res. 2006;13:385-309.

4. Matsui K, Kita Y, Ueshima H. Informed consent, participation, and wirthdrawal from a population based cohort study involving genetic analysis. J Med Ethics. 2005;31:385-392.

5. Emmanuel E, Wendler D, Grady C. What makes clinical research ethical? JAMA. 2000;283:2701-2711.

6. Edwards S. Research participation and the right to withdraw. Bioethics. 2005;19(2):112-130.

7. Frank G. Current challenges in clinical trial patient recruitment and enrollment. SoCRA Source. 2004 Feb;30-38.

8. Ye C. Data withdrawal in randomized controlled trials: defining the problem and proposing solutions. A commentary. Contemp Clin Trials. Epub 2011 Feb 4.
9. Dow A, Goldstein W. FDA and OHRP guidance on data retention and other considerations applicable to subjects who withdraw from clinical research. Available from: http://www.ebglaw.com/files/24854 CLIENT\%20ALERT-FDA\%20and\%20OHRP\%20Guidance \%20 on\%20Data\%20Retention.pdf. Accessed March 16, 2011.

10. Keller C, Gonzales A, Fleuriket K. Retention of minority participants in clinical research studies. West J Nurs Res. 2005;27(3):292-306.

11. Office for Human Research Protections (OHRP). Draft Guidance on Important Considerations for When Participation of Human Subjects in Research is Discontinued. 2010 Nov. Available from: http://www.hhs. gov/ohrp/requests/200811guidance.pdf. Accessed March 16, 2011.

12. National Institutes of Health. Code of Federal Regulations Title 45 Part 46: Basic HHS Policy for the Protection of Human Subjects. Available from: http://ohsr.od.nih.gov/guidelines/45cfr46.html. Accessed March $16,2011$.

13. US Food and Drug Administration. Code of Federal Regulations Title 21 Part 50: Protection of Human Subjects. Available from: http://www.accessdata.fda.gov/scripts/cdrh/cfdocs/cfcfr/CFRSearch cfm?CFRPart=50\&s howFR=1. Accessed March 16, 2011.
Open Access Journal of Clinical Trials

\section{Publish your work in this journal}

The Open Access Journal of Clinical Trials is an international, peerreviewed, open access journal publishing original research, reports, editorials, reviews and commentaries on all aspects of clinical trial design, management, legal, ethical and regulatory issues, case record form design, data collection, quality assurance and data auditing

Submit your manuscript here: http://www.dovepress.com/open-access-journal-of-clinical-trials-journal

\section{Dovepress}

methodologies. The manuscript management system is completely online and includes a very quick and fair peer-review system, which is all easy to use. Visit http://www.dovepress.com/testimonials.php to read real quotes from published authors. 